\title{
DUST DRIVEN MASS LOSS FROM PULSATING AGB-STARS *
}

\author{
E.A. DORFI, M.U. FEUCHTINGER, S. HÖFNER \\ Institut für Astronomie \\ A-1180 Wien, Austria
}

ABSTRACT. A new numerical method allows an acurate calculation of the radiation hydrodynamics of time dependent stellar winds including also the radiation pressure on newly formed dust grains. The numerical procedure is based on an adaptive grid which distributes the grid points at locations of large gradients. All equations are written in conservation form and a monotonic 2 . order transport scheme is used to advect the physical variables through the cell boundaries. We are able to resolve the shock waves running through the stellar atmospheres. These waves are generated by a pulsating star which is simulated by a moving piston. The following plots show the radial velocity and temperature structure of an extended atmosphere and several shock waves are clearly seen. Note that the innermost shock waves is a so-called supercritical shock where the radiative cooling zone behind the wave is clearly visible. The outer waves are almost isothermal because the material is optically thin in this region. The stellar parameters of this example are given by $M=1.2 M_{\odot}, L=5315 L_{\odot}$ and $R=270 R_{\odot}$ and the period of the moving piston is fixed at 350 days yielding a massive and slow wind with a mass loss rate of $\dot{M}=1.2410^{-6} M_{\odot} \mathrm{yr}^{-1}$ and a final velocity of $v=7.7 \mathrm{~km} \mathrm{~s}^{-1}$.

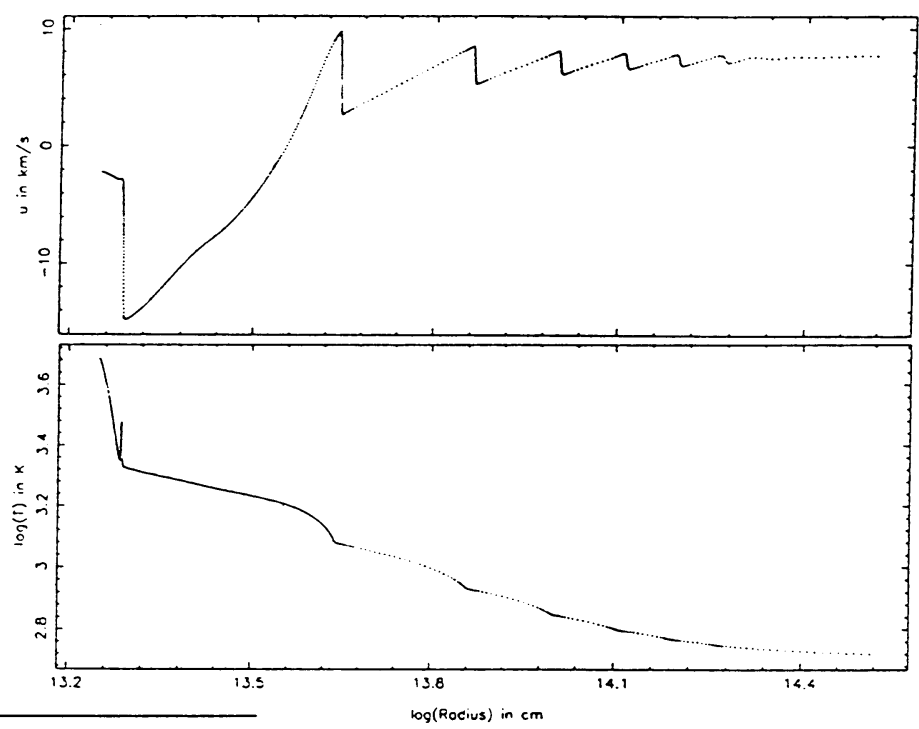

* supported by the FFWF under project number P8411 